\title{
Glorifier le royaume et le souverain en temps de crise : émotions et royauté chez Christine de Pizan, les chroniqueurs de Charles VI, et Pierre de Ronsard
}

\author{
Glorifying the king and the kingdom in times of crisis: \\ emotions and kingship in the works of Christine de Pizan, \\ Royal chroniclers, and Pierre de Ronsard
}

\author{
Charles-Louis Morand Métivier \\ The University of Vermont \\ cmorandm@uvm.edu
}

\begin{abstract}
This article proposes to explore crises from seemingly different backgrounds and periods (the madness of Charles VI of France and the French Wars of Religion) to demonstrate how these elements were used by authors in order to create emotional narratives in support of the failing monarchy. In the two situations, the French community was constructed as an entity which duty was to support the king. However, the authors use different methods to transmit their messages. While Christine de Pizan and the chroniclers advocate for the building of an integrating, all encompassing community around the love of and for the king, Ronsard's France is tasked at differentiating the Catholics and the Protestants, in an effort to construct France also as a monarchy loving community, of which the protestants are presented as the enemies.
\end{abstract}

Keywords: Christine de Pizan, Pierre de Ronsard, chronicles, wars, religion, emotions, community, France

Littérature et politique sont, au Moyen Âge et à la Renaissance, étroitement liés, si bien que beaucoup d'auteurs ont laissé une trace indélébile dans l'histoire, notamment grâce à la façon dont ils ont commenté les événements de leur temps. Christine de Pizan (1364-1430) et Pierre de Ronsard (1524-1585) ont été, chacun à leur époque, des figures-clefs de la cour. Tous deux ont côtoyé de très près les 
souverains de leur époque et ont donc été parmi les témoins les plus proches des actions de ces derniers en faveur du royaume, mais également de la façon dont les vicissitudes historiques ont impacté leurs vies. Les règnes de ces différents souverains ont été marqués par des évènements qui ont durement éprouvé le royaume, allant jusqu'à ébranler les fondations mêmes de la France. Christine de Pizan a ainsi été le témoin direct de la folie de Charles VI, qui a conduit le royaume à se déchirer au cours d'une sanglante guerre civile, alors même que des forces extérieures mettaient déjà en péril son intégrité ${ }^{1}$. Ronsard, de son côté, a assisté à l'arrivée de la Réforme en France, et a particulièrement été marqué par les premières guerres de religion (voir Holt, 2002).

Il pourrait paraître incongru, au premier abord, de rapprocher deux auteurs aussi différents que Ronsard et Christine. Cependant, malgré les différentes époques dans lesquelles ils vécurent, leurs œuvres s'inscrivent dans un schéma politique et intellectuel qui présente bien des points communs. Ronsard et Christine avaient tous les deux un profond attachement, que je qualifierais même d'amour, pour le royaume ; de même, tous deux avaient une position cruciale à la cour, que ce soit en tant que proche du roi (Christine) ou poète officiel (Ronsard) ; Christine de Pizan a vécu toute sa jeunesse auprès de la cour de Charles $\mathrm{V}$, son père Thomas de Pizan ayant été mandé par le roi afin de devenir son astrologue ainsi que l'un de ses plus proches conseillers. Pierre de Ronsard avait été reçu à la cour et était personnellement soutenu dans son projet de Franciade par Henri II ; sa position à la cour ne fit que se renforcer avec les souverains et régents suivants. Cette présence quasi continuelle auprès des souverains de leur époque a eu une importance primordiale dans leur développement personnel, professionnel et intellectuel. Tous deux ont ainsi été aux premières loges de deux des plus grandes crises politiques de leur temps : la folie du roi Charles VI et la première des guerres de religion. En tant qu'acteurs de la cour et donc de témoins privilégiés des événements tragiques de leur époque, c'est donc naturellement qu'ils ont tous deux distillé une relation émotionnelle aux problèmes du royaume dans leur travail, même si leurs œuvres ne sont pas de même nature (Pizan a écrit des œuvres allégoriques et didactiques, alors que Ronsard a écrit de la poésie amoureuse et des œuvres encomiastiques). Les œuvres qu'ils ont écrites dans des climats de crise sont donc profondément marquées par la nécessité de redonner sa grandeur et sa force à un royaume menacé. Cependant, les deux auteurs en proposent une vision drastiquement différente. Pizan propose un schéma dans lequel l'amour (du roi, du peuple) permet l'unification de tous autour d'une entité étatique forte. Chez Ronsard, même si l'amour du souverain et de la religion sont au cœur de la défense du royaume, elle se mélange à la colère, voire à la haine du protestant, qui est accusé de vouloir détruire les fondations du royaume que Ronsard a construites dans son œuvre.

\footnotetext{
${ }^{1}$ Pour un panorama général du règne de Charles VI, voir Autrand (1986).
} 
Comment penser l'idée de communauté, et particulièrement sa construction par le biais des émotions ? Ces questions ont été déjà abordées dans de nombreuses études. Émile Durkheim, dans Les Formes élémentaires de la vie religieuse (1912) développe la notion d'effervescence collective. Il appuie ce concept sur le principe que, dans un groupe sociétal, certains éléments et moments particuliers permettent à un groupe de se regrouper autour de grands concepts et principes, s'unifiant alors au-delà de tout clivage. Si Durkheim est parti du postulat que la religion était l'élément déclencheur de cette action, cette théorie a été récemment utilisée dans des contextes différents, notamment celui du rôle des émotions dans la construction d'une entité nationale unificatrice (voir notamment Beyer \& al., 2014).

L'idée de l'émotion en tant qu'instrument communautaire est également centrale aux travaux de Barbara Rosenwein, qui a créé l'idée de communautés émotionnelles. Rosenwein explique que ces communautés se construisent autour d'individus pouvant venir d'horizons divers, mais qui ont la particularité d'adhérer aux mêmes contextes émotionnels, en acceptant ou rejetant les mêmes idées et émotions. Ces communautés ne sont pas inclusives, et plusieurs d'entre elles peuvent coexister dans un contexte donné. Finalement, et c'est le point sur lequel cette théorie peut être mise en parallèle avec les idées de Durkheim, ces communautés émotionnelles ont le potentiel d'être protéiformes, tant au niveau de leurs constituants qu'au niveau de la population qu'elles veulent et peuvent englober. Ainsi, ces communautés peuvent être aussi petites qu'une taverne, ou bien atteindre des dimensions beaucoup plus importantes par exemple celle d'une paroisse. Mon approche dans cet article est basée sur ces deux idées. Je veux me focaliser sur la façon dont les Pizan et Ronsard défendent dans leurs écrits deux idées différentes de ce que le royaume devrait être, afin de sortir du marasme et de la crise dans laquelle il est plongé.

\section{CHRISTINE DE PIZAN ET LA GLORIFICATION DE LA FONCTION ROYALE}

Christine de Pizan était l'un des auteurs médiévaux les plus importants; ses œuvres sont un témoignage vivant de la politique française de la fin du XIV ${ }^{\mathrm{e}}$ siècle et du début du $X V^{\mathrm{e} 2}$. Elle a encensé le roi Charles $\mathrm{V}$ dans de nombreuses œuvres. Cette affection s'est transférée, pour ainsi dire, vers Charles VI à la mort de son père. Sa glorification du roi est double. Elle est bien sûr un ardent défenseur du roi en tant qu'être humain ; cependant, c'est également l'essence royale qu'elle porte aux nues, selon le principe des deux corps du roi, procédé qu'Ernst Kantorowicz (1989) a démontré par le passé. En effet, Christine fait de nombreuses références

\footnotetext{
${ }^{2}$ La littérature critique sur l'œuvre politique de Christine de Pizan est abondante ; on pourrait citer, par exemple : Autrand (2009), Blumenfeld-Kosinski (2003) et Adams (2014).
} 
à l'idée de généalogie, montrant à quel point les rois - passés et présents - sont issus d'une même branche, qui se reproduit et qui se transmet : « [...] me semble qu'assez appertient parler aucunement en nostre traictié du beau fruit et belles branches de l'arbre dont cy devant avons ja parlé de la racine [...] ce est le sage roy, Charles, dont sailli fruit bel et bon [...] ce sont ses tres dignes et haulz enfens » (Christine de Pizan, 2009, p. 123). La transmission du sang royal entre père et fils transmet ainsi la grandeur de l'institution royale. Quand bien même le roi connaitrait des problèmes au cours de son règne - comme cela était le cas avec Charles VI - il est automatiquement «protégé » chez Christine par son ascendance glorieuse qui fait de lui l'héritier de la grandeur des rois de France passés. De même - et c'est l'exemple que l'on peut tirer du Livre de paix - la descendance de Charles VI aura hérité de cette grandeur ancestrale, et n'aura donc pas à souffrir des problèmes qui auront émaillé le règne de leur aïeul. J'ai déjà analysé la relation émotionnelle entre Charles $\mathrm{V}$ et Christine de Pizan, en expliquant comment celle-ci créait une image d'un roi émotionnel, aimant et étant aimé, afin de rassembler le royaume autour de sa personne et de son pouvoir (voir Morand Métivier, 2017). Ce travail a toujours été central à l'œuvre de Christine, et elle l'a continué de façon encore plus avancée avec sa construction émotionnelle de Charles VI.

Beaucoup des œuvres de Christine font allusion à Charles VI, même celles qui ne sont pas à première vue encomiastiques. Ainsi, le Livre de la mutacion de Fortune, où Christine narre son allégorique transformation en homme, explique la façon dont il a été durement marqué par la Fortune, à la manière d'autres souverains :

[...] Charles le Vi. ${ }^{\text {, }}$

Couronné, en gloire haultiesme,

En aage de.XII. ans, je vy,

Et moult eust esté assouvy

Prince en toute grace, sanz doubte,

Se Fortune, qui n'y voist goutte,

Ne l'eust grevé de maladie,

Mais nonobstant quoy que nul dye,

(Dieu mercis !) onques plus en paix

Le regne ne fu, n'onques maiz

Plus deschié des ennemis

Qui par long temps ont peine mis

A le grever, n'onques encore

Le pueple mieulx n'obeÿ que ore

Fait a leur prince [...]. (Christine de Pizan, 1966, p. 77, vv. 23545-560)

Même dans une période difficile, où Fortune l'accable, Christine souligne le fait que, malgré la maladie, le roi tient son rôle avec courage. Le Charles $\mathrm{V}$ émotionnel qu'elle a mis en avant dans ses œuvres fait écho au Charles VI bon, aimé et aimant qu'elle souhaite également mettre en avant. Charles VI est construit comme un roi 
fort, mais qui tire sa grandeur de son rapport émotionnel aux autres, et de son courage face à l'adversité.

Chez Christine, Charles VI ne se contente pas d'être un roi suivant le canon monarchique (sagesse, intelligence, etc.). Il est également un roi émotionnel et émotif, ce que Christine considère comme crucial et nécessaire à l'accomplissement de sa fonction. Le Livre de paix se focalise sur le sujet mentionné dans son titre, à savoir le devoir d'un souverain de promouvoir et d'atteindre la paix qui, dans le contexte particulier de Charles VI, était protéiforme, au vu des nombreux conflits qui minaient le royaume ${ }^{3}$. Ce livre, dédié au Dauphin, met également en avant un élément fondamental du développement royal. Si Charles VI, malgré sa maladie, est un roi bon, c'est qu'il a hérité de ces qualités. De ce fait, Christine effectue un véritable appel à la généalogie royale. Son père et son grand-père sont érigés en exemples que le futur roi se doit de suivre afin d'être un aussi bon souverain qu'eux.

Christine souligne que les vertus cardinales d'un souverain doivent être « la vertu de clemence et benignité » (Christine de Pizan, 2008, p. 257). Charles VI est présenté comme un exemple de ce genre de souverain par Christine : " tesmoing ton bon pere le roy charles VI, qui a present regne » (Christine de Pizan, 2008, p. 258). La circulation constante qui est faite entre le grand - père, le fils et le petit-fils met en avant une identité commune qui permet d'expliquer comment cette relation doit être transférée envers les souverains qui suivent. La première communauté émotionnelle qui se construit, chez Christine, est donc basée sur l'idée d'amour familial et d'amour du peuple. Dans le Livre des faits du bon roi Charles $V$, elle a développé de façon très poussée la façon dont le roi était un homme aimé et aimant; dans le Livre de paix, elle met cette fois en relation ces émotions entre le roi et son fils. Charles $\mathrm{V}$ est souvent représenté par Christine comme étant l'apex de la royauté ; cette perfection se propage également dans sa descendance. C'est pour cela que le sentiment d'amour entre le roi et son peuple est comparable à celui qui unirait une famille parfaite ; la maladie du roi, loin d'être un frein, apparaît comme une autre épreuve qui permet de resserrer les liens entre le roi et les membres du corps politique, créant alors une communauté royale englobant tous ceux qui ressentent cet amour.

Si Christine est un des grands témoins du règne de Charles VI, ces événements ont été très fidèlement retranscrits par de nombreux chroniqueurs, comme dans la Chronique du Religieux de Saint-Denis et l'Histoire de Charles VI de Jean Juvénal des Ursins. Ces œuvres n'omettent aucun détail, comme la folie du roi, et ne se censurent absolument pas; dans son introduction à la réédition de la Chronique $d u R e$ ligieux de Saint-Denis, Bernard Guenée explique que le but même de ces chroniques était de montrer les évènements avec le plus de vérité possible, afin de pouvoir devenir des témoins des règnes. La folie du roi est particulièrement intéressante

\footnotetext{
${ }^{3}$ Le règne du roi a été traversé par la guerre de Cent ans, le grand Schisme d'Occident, et la guerre civile entre Armagnacs et Bourguignons.
} 
à prendre en considération par rapport à son ressenti émotionnel à la cour. Juvénal des Ursins indique que cet événement à été profondément traumatisant pour les suiveurs du roi : «Tout le peuple pleuroit et gemissoit, et en cet estat le voyoit chacun qui vouloit. Des Anglois mesmes par le moyen du seigneur de La Riviere le vinrent voir [...] La chose vint à la congnoissance du pape et du roy d'Angleterre, qui en furent très-desplaisans. » (1841, p. 377). Le Religieux de Saint-Denis suit le même schéma :

Cette nouvelle plongea toute la cour dans la désolation. Les seigneurs atterrés de ce malheur témoignaient leur affliction par leurs larmes et leurs cris ; ils prenaient des habits de deuil, et donnaient toutes les marques d'un sérieux désespoir. [...] les ambassadeurs du roi d'Angleterre vinrent comme les autres à ce triste spectacle. [...] Le roi d'Angleterre, ainsi que me l'ont assuré des témoins dignes de foi, apprit avec douleur l'infortune du roi Charles. (Chronique, 1994, p. 23)

Dans ces passages, où j'ai mis en italiques les marqueurs émotionnels, l'état du roi touche profondément ses suiveurs. Le peuple lui-même aimait profondément son souverain. Dans le Livre de paix, le passage d'une entrée du roi dans une ville est relaté comme un échange entre le souverain et ses sujets. À la vue du cortège royal, ces dernieres se pressent pour essayer de l'apercevoir : « Lequel est le roy? Lequel est le roy? Je les vois tous ensemble ». Charles V, en souverain aimant et se sachant aimé, veut aussi répondre aux attentes de son peuple: "Si aloit la gent qui a l'encontre de lui de toute pars fuioient, si comme le bon peuple de France a coustume de faire vers leur seigneur, et quant grant presse y avoit en quelque grant place, en signe d'amour et benignité recevant le salu de tant de gent, il ostoit son chapel » (Christine de Pizan, 2008, p. 215).

Tout comme Christine l'a fait, les chroniqueurs notent bien l'impact que le roi avait sur sa population, et les liens d'amour qui unissait les uns et les autres. De même, lorsque Charles VI arrive à recouvrer la santé lors de brefs moments de lucidité, ses premières actions sont dirigées vers le royaume, qu'il veut diriger de son mieux, dans une évidente démonstration d'amour. Les chroniqueurs détaillent les différents passages de lucidité du roi comme un regain de sa fonction royale, pendant lesquelles la perfection monarchique (de gouvernance et de piété) que Christine met en valeur est bien présente. Juvénal des Ursins explique qu'en 1399, « le roy retourna en santé, et avoit bon sens et entendement, et fit la solemnité de Pasques en son hotel a Sainct-Paul [...] le roy receut le sainct sacrament de confirmation, en grande devotion » (Jean Juvénal des Ursins, 1841, p. 377). En 1405, «Quand le roy revint à aucune convalescence, il prit les choses en sa main » (Jean Juvénal des Ursins, 1841, p. 424). Sa mort en 1422 peina grandement le peuple : «En son temps il fut piteux, doux et benin à son people, servant et aimant Dieu, et grant aumonier » (Jean Juvénal des Ursins, 1841, p. 572). Le chroniqueur de Saint-Denis suit le même modèle : «Le roi lui-même, suivant le désir qu'il en avait manifesté plusieurs fois, 
se rendit à cheval [...] à l'église de Saint-Denis, et fit une visite au patron particulier de la France » (Chronique, 1994, p. 93).

Le roi Charles VI, malgré les terribles événements qui ont entaché son règne, n'est pas diminué par les nombreuses références qui sont faites à sa maladie. Au contraire, il est construit comme un roi résiliant, qui utilise le moindre moment de lucidité pour se donner complètement à son royaume. Que ce soit chez Christine ou chez les chroniqueurs, le roi ne peut être détaché d'une image émotionnelle forte, puisque malgré l'adversité, il se donne corps et âme à son pays. Il est au centre d'une France parfaite, regroupée autour de lui comme une famille afin de pouvoir traverser ces difficiles moments.

\section{RONSARD : UNE RHÉTORIQUE DU REJET}

La France dans laquelle Ronsard écrit était déchirée par des oppositions dogmatiques. Le roi de France était officiellement un « Rex Christianissimus », un « Roi Très-Chrétien » dont la défense de la foi était un important objectif. Aimer son peuple, et donc se faire aimer par le roi, dans cette époque de troubles, sous entendait l'adhésion à la religion du souverain. L'idée même de religion était inscrite au cœur même de la France. La Réforme, dès lors, était perçue comme une volonté délibérée de se détacher de l'ensemble parfait et immuable de la royauté. Du côté protestant, la seule volonté présente est celle de vouloir pratiquer sa foi librement et pacifiquement, sans risquer des exactions ou représailles ${ }^{4}$. Cependant, comme l'indique Arlette Jouanna, aucun des deux camps ne pouvait se satisfaire d'une situation qui était vue comme un ébranlement complet de la composition du royaume telle qu'elle était connue à ce moment (2013, p. 174).

Ronsard était ce que je désignerais comme un défenseur extrémiste de la couronne ; sa poésie encomiastique se nourrit du système qu'elle défend et idolâtre. Le royaume est l'héritier d'une grandeur éternelle et ancestrale, qui est mise à mal par les remises en question protestantes ${ }^{5}$. L'attaque de Ronsard est ainsi principalement dirigée contre les protestants non pas, dans un premier temps, parce que leur dogme est considéré comme impie, mais avant tout parce qu'ils sont ce que je désignerais comme des « disrupteurs » de tradition. Géralde Nakam explique qu'elle est chez Ronsard «le pilier de son idéologie : maintien, sur le plan politique, de la hiérarchie sociale, monarchique et catholique, transmission, sur le plan culturel, du patrimoine poétique » (2005, p. 78). L'attaque des protestants chez Ronsard est protéiforme. Il met en avant le fait que ces derniers font souffrir le royaume, et se présente

\footnotetext{
${ }^{4}$ Voir notamment «Epistre au Roy de France François I ${ }^{\mathrm{er}} »($ Calvin, 2008, vol. 1, pp. 115-80).

${ }^{5}$ Ronsard essaiera dans sa Franciade de retracer les origines éternelles de la France, un travail titanesque qui restera inachevé.
} 
comme le défenseur du royaume dans la Continuation : "Madame, je serois ou du plomb ou du bois, / Si moy, que la nature a fait naistre François, / Aux siècles advenir je ne contois la peine / Et l'extreme malheur dont nostre France est pleine » (Ronsard, 1979, p. 81, vv. 1-4). Là où les choses étaient parfaites avant, soulignées par des idées de puissance et de beauté, la Réforme a transformé le paysage idéal de la France en une terre faible, pleine de doutes, et qui jette le discrédit sur ce passé glorieux. L'attaque de Ronsard prend corps à travers la dénonciation de la Réforme en tant qu'acte social bouleversant la perfection émotionnelle du royaume. C'est dont le côté « anarchique » de la Réforme que Ronsard s'attache à combattre.

L'engagement politique et émotionnel de Ronsard est une volonté de redonner sa grandeur au royaume, que François Rigolot explique en disant que « le but de la littérature engagée est plutôt de remettre de la cohérence dans une France traversée par les forces séparatrices de la rébellion » $(1989$, p. 65). Les bases émotionnelles du passé du royaume sont prises en exemple ; il faut que celui-ci arrive à retrouver l'amour et la fierté de son peuple ${ }^{6}$. Dans cet esprit, l'ire de Ronsard est le ciment non pas forcément de la France, mais de $s a$ France, celle qu'il a construite dans ses œuvres. Le royaume qu'il crée dans sa poésie est une entité parfaite, qui ne connaît de problème, et qui est unie avec fierté derrière la famille royale. Pour Ronsard, donc, il n'est pas possible d'imaginer un royaume dont la population ne soit pas pleinement et entièrement dévouée. Il effectue une construction émotionnelle en opposition entre les deux groupes. Dans un premier temps, il met en exergue la fausseté des « étrangers » qui veulent détruire le royaume ; ensuite, il critique les intellectuels réformés qui attaquent directement son entreprise de glorification du royaume et sa personnalité de poète. Sur ces deux fronts, les éléments offensifs et défensifs de Ronsard tournent autour de l'utilisation de la colère, qui permet d'attaquer les éléments dissidents du royaume, ainsi que les opposants à son art. Ronsard est le dépositaire autoproclamé de la postérité du royaume, de sa grandeur perdue que ses ennemis veulent détruire; les Discours des misères de ce temps débordent littéralement du champ lexical de la tristesse et de la désolation, associés à l'irrémédiable déclin du royaume ${ }^{7}$. La Terre et les cieux eux-mêmes sont témoins

\footnotetext{
${ }^{6}$ Voir notamment dans l'intégralité des œuvres des Discours les innombrables références au passé mythique et glorieux de la France.

${ }^{7}$ Relever l'intégralité du vocabulaire émotionnel de Ronsard s'avèrerait beaucoup trop long pour un travail de ce genre. Cependant, un passage particulier met en relief la tristesse de Ronsard, mêlée à la mise en relief des malheurs à venir :

O toy historien, qui d'ancre non menteuse

Escrits de nostre temps l'histoire monstrueuse,

Raconte à nos enfans tout ce malheur fatal,

Afin qu'en te lisant ils pleurent nostre mal

Et qu'ils prennent exemple aux pechés de leurs pères,

De peur de ne tomber en pareilles misères (1979, p. 76, vv. 115-120).

J'ai moi-même mis en italiques les termes liés au champ lexical de la tristesse et du regret.
} 
de la tristesse générale de la situation : «Le Ciel qui a pleuré tout le long de l'année, / Et Seine qui couroit d'une vague éfrenée, / Et bestail et pasteurs largement ravissoit, / De son malheur futur Paris avertissoit » (Ronsard, 1979, p. 70, vv. 107-110).

Ce qui caractérise la Réforme chez Ronsard, c'est qu'elle est une menace étrangère ; elle apporte peur et déséquilibre émotionnel dans le royaume. Il voit en elle avant toute autre chose une force étrangère qui essaie d'envahir la France pour la détruire de l'intérieur. Il la construit ainsi sous la forme d'une communauté émotionnelle maléfique. Dans la Remontrance, il pousse cette analogie en la rendant l'égale des grands envahisseurs du passé : «Je n'aime point ces noms qui sont finis en os. / Gots, Cagots, Austregots, Visigots et Huguenots / Ils me sont odieux comme la peste, et je pense / Qu'ils sont prodigieux à l'empire de France » (Ronsard, 1979, p. 101, vv. 217-20). Ce qui insupporte Ronsard, c'est l'attaque de ceux qu'il considère comme des barbares contre le royaume. Cette énumération associe les huguenots avec les grands envahisseurs Goths qui terrorisèrent le royaume de France aux $\mathrm{IV}^{\mathrm{e}}$ et $\mathrm{V}^{\mathrm{e}}$ siècles. Ils ont pour ainsi dire le même nom, sont détestés du poète et ne sont occupés qu'à piller et détruire. Par ces quelques vers, Ronsard pose la Réforme comme une entité négative et destructrice, dont l'objectif ne peut être que néfaste, et contre laquelle seulement la même approche que la sienne - la haine - est acceptable.

Ronsard associe rapidement attaque contre le royaume avec une attaque contre sa personne dans la Responce, probablement l'une des pièces les plus virulentes qu'il ait écrites. Il met en avant le fait que les écrits qui l'attaquent, quels qu'ils soient, sont avant tout également des attaques contre la France, puisqu'il n'a jamais arrêté de clamer son amour et son attachement envers elle. Ce thème va devenir le paradigme central de la Responce : «Tu mesdis de mon nom que la France renomme, / Abboyant ma vertu, et faisant du bragard, / Pour te mettre en honneur tu te prends à Ronsard » (Ronsard, 1979, p. 122, vv. 4-6). Le poète souligne l'arrogance («bragard ») de ses accusateurs, en se positionnant comme un membre important, voire nécessaire, à la France. Il faut voir chez Ronsard une sacralisation - qui ne se revendique pas comme pédante - de sa personne et de ce qu'elle représente par rapport à la personnalité de la France. Daniel Ménager explique que « réfuter un discours, c'est donc en même temps réfuter une culture et une idéologie ; réfuter une histoire aussi qui met ce discours sur les lèvres de celui qui parle » (Ménager, 1979, p. 262). Je vais aller plus loin, en insinuant également que se moquer de Ronsard, c'est pour lui réfuter l'association émotionnelle qu'il a érigée entre religions, royauté et luimême ; c'est donc également réfuter l'existence de la communauté émotionnelle de la France qu'il crée. Il érige sa personne et son écriture comme faisant partie à part entière de la communauté nationale ; de même qu'il fait partie du royaume, il « est » également le royaume. Dans ce sentiment national exacerbé, Ronsard implique encore davantage son appartenance religieuse comme un élément primordial constitutif de cette communauté qu'il défend. 
L'une des attaques les plus fortes effectuées contre Ronsard consistait à le critiquer pour la très haute idée de lui-même qu'il laissait transparaître, et par là même induire que Ronsard utilisait son amour de la France afin de servir ses propres ambitions de gloire. La Responce critique ces attaques en utilisant pourtant le même artifice, à savoir que Ronsard s'impose comme le plus grand poète de la France, tout en expliquant que ces attaques n'en sont pas vraiment, puisque ce qu'il dit est vrai. Francis Higman souligne le fait que « in his Responce, while continuing the arguments about religious crisis, Ronsard resolves to refute the criticisms of his poetic powers by giving a demonstration of those powers. He is deliberately showing off » (1998, p. 483). Il ne faut cependant pas voir cette attaque, dans l'esprit de Ronsard, comme une simple défense de ce qu'il considère comme la supériorité évidente de son art, mais plutôt comme la marche ultime de sa défense de la France et de son assimilation à cette dernière, par laquelle il devient véritablement cette idée de la France qu'il a défendue tout au long de ses Discours.

Comme il l'a fait précédemment, Ronsard souligne l'importance historique de son œuvre, destinée à souligner la nécessité de préparer l'avenir du royaume. Ronsard s'inscrit comme le guide de la France de l'avenir, quand il explique « Mais avant que finir, entend Race future, / Et comme un testament garde ceste ecriture » (Ronsard, 1979, p. 153, vv. 1163-64). Le double-entendre sur le mot écriture ramène alors les lecteurs à la Bible, le livre de base de la chrétienté. Ronsard souhaite alors que ses écrits deviennent le point de départ d'une communauté française partageant les mêmes points de vue que lui. Le Discours est alors la Sainte Écriture de la nouvelle communauté émotionnelle de la France, qui doit adhérer au guide de vie que Ronsard a voulu instaurer. Le moment le plus paroxystique de la Responce est celui où Ronsard se confond à la France, ou plutôt où il devient la France, cette communauté émotionnelle qu'il a longtemps défendue contre les attaques nombreuses des huguenots. La France que Ronsard défend, si elle est celle de Charles IX, une France forte et catholique, unie autour de son roi, et cherchant à se débarrasser ou à assimiler la menace genevoise, c'est avant tout sa France, qu'il a imaginée dans sa poésie, et qu'il a mise en action.

Ronsard crée sa France à l'intersection de sa poésie, de son amour et respect de la religion catholique et de la monarchie, et de la défense contre les protestants qu'il effectue. La France de Ronsard est donc une France du combat, de la résistance qui s'organise autour d'un père poétique qui transcende les aspects monarchiques et religieux de la communauté afin de les modeler à son image idéale, celle d'un amour inconditionnel de la France telle qu'il la conçoit. Elle est modelée autour d'un développement de l'intellect et d'une religion communautaire, dans laquelle il s'exécute en tant que poète national, c'est-à-dire en tant que scribe de la grandeur du pays. Cette entité émotionnelle, qui doit pour le poète se calquer sur le modèle qu'il a défini, est ainsi terriblement restrictive et exclusive ; quiconque ne se moule pas parfaitement dans ce carcan idéologique et religieux qui a été mis en place par Ron- 
sard est donc de facto exclu de sa France. Cette communauté émotionnelle est basée sur l'amour des belles lettres en tant qu'instrumentalisation de l'esprit français, sur l'amour du roi et de la religion. Elle est également basée sur la haine et la colère envers les protestants, à cause de leur non-acceptation de ces règles intransigeantes dans l'esprit du poète.

Les réponses émotionnelles à ces deux crises institutionnelles sont très différentes chez les deux auteurs. Cependant, chez Ronsard et Christine, on ressent bien la nécessité de créer du lien littéraire afin de donner une réponse émotionnelle à de graves crises politiques, dans lesquelles le tissu même du royaume est mis en danger. Entre union et exclusion, entre amour et colère, les deux auteurs démontrent l'infinité complexité de la construction communautaire, du royaume, s'appuyant sur des ressorts opposés (unification complète de la population vs. exclusion de groupes dissidents) ; ils soulignent également à quel point le même objectif, celui de défendre la France, mène à des solutions aux antipodes l'une de l'autre. 


\section{BIBLIOGRAPHIE}

Adams, T. (2014). Christine de Pizan and the Fight for France. University Park: Penn State University Press.

Autrand, F. (1986). Charles VI. Paris : Fayard.

Autrand, F. (2009). Christine de Pizan. Paris : Fayard.

Beyer, M. \& al. (2014). The social consequences of collective emotions: national identification, solidarity and out-group derogation. URL : http://www.polsoz.fu-berlin.de/soziologie/arbeits bereiche/emotionen/team/Professur/preprints/Beyer_etal_SocialConsequencesCollectiveEmo tions_preprint.pdf?1392642926

Blumenfeld-Kosinski, R. (2003). Christine de Pizan and the Political Life in Late Medieval France. In B. K. Altman, D. L McGrady (Eds.), Christine de Pizan. A Casebook (pp. 9-24). New York - London : Routledge.

Calvin, J. (2008). Institution de la religion chrétienne. 1541 (vols. 1-2). O. Millet (Ed.). Genève : Droz.

Christine de Pizan (1966). Le Livre de la mutacion de Fortune (vol. IV). S. Solente (Ed.). Paris : Picard.

Christine de Pizan (2008). The Book of Peace. K. Green, C. J. Mews, J. Pinder (Eds.). University Park : Penn State University Press.

Christine de Pizan (2009). Le Livre des fais et bonnes meurs du sage roy Charles V. N. Desgrugillers-Billard (Ed.). Clermont Ferrand : Éditions Paléo.

Chronique du religieux de Saint-Denis (1994) (vol. I). B. Guenée (Ed.). M. L Bellaguet (Trans.). Paris : Éditions du comité de travaux historiques et scientifiques.

Higman, F. (1998). Lire et découvrir : la circulation des idées au temps de la Réforme. Genève : Droz.

Holt, M. P. (2002). Renaissance and Reformation France, 1500-1648. Oxford : Oxford University Press.

Jean Juvénal des Ursins (1841). Histoire de Charles VI, roy de France. In J.-A.-C. Buchon (Ed.), Choix de chroniques et mémoires sur l'histoire de France avec notices littéraires (pp. 323-537). Paris : A. Desrez. URL : https://archive.org/details/histoiredecharle00juv

Jouanna, A. (2013). Le Pouvoir absolu. Naissance de l'imaginaire politique de la royauté. Paris : Gallimard.

Kantorowicz, E. (1989). Les Deux corps du roi (J. P. Genet, N. Genet, Trans.). Paris : Gallimard.

Ménager, D. (1979). Ronsard, le roi, le poète et les hommes. Genève : Droz.

Morand Métivier, Ch.-L. (2017). Émotions politiques, politiques émotionnelles : Christine de Pizan et Charles V. The French Review, 90 (3), 13-24.

Nakam, G. (2005). Chemins de la Renaissance. Paris : Honoré Champion.

Rigolot, F. (1989). Poétique et politique : Ronsard et Montaigne devant les troubles de leur temps. In M. Dassonville (Ed.), Ronsard et Montaigne, écrivains engagés ? (pp. 57-70). Lexington : French Forum.

Ronsard, P. de (1979). Discours. Derniers Vers (Y. Bellanger, Ed.). Paris : GF Flammarion.

Rosenwein, B. (2006). Emotional Communities in the Early Middle Ages. Ithaca : Cornell University Press. 